\title{
USO DA ACUPUNTURA NA PREVENÇÃO DE ARRITMIAS CARDÍACAS EM RATOS WISTAR
}

\author{
Mariana Paz Rodrigues ${ }^{1}$, Alessandra Melchert ${ }^{2}$, Tales Dias do Prado ${ }^{3}$ \\ ${ }^{1}$ Professora Mestre da Universidade de Rio Verde - UniRV, Rio Verde, Goiás. \\ ${ }^{2}$ Professor Assistente doutor da Universidade Estadual Paulista Júlio de Mesquita Filho - UNESP, \\ Botucatu - SP. \\ ${ }^{3}$ Professor Adjunto Mestre do Departamento de Cirurgia Veterinária da Universidade de Rio Verde - \\ UniRV, Rio Verde, Goiás. \\ E-mail: mah paz@hotmail.com
}

Recebido em: 08/09/2015 - Aprovado em: 14/11/2015 - Publicado em: 01/12/2015 DOI: http://dx.doi.org/10.18677/Enciclopedia_Biosfera_2015_238

\begin{abstract}
RESUMO
O presente estudo visa avaliar o efeito da acupuntura na prevenção de arritmias induzidas por doses crescentes de adrenalina em ratos. Foram utilizados 26 ratos Wistar, saudáveis, machos, divididos em 2 grupos: Grupo 1 (G1) - acupuntura durante 30 minutos, nos pontos Pericárdio 6 (Pc6) e Coração 7 (C7), antes da administração de adrenalina por via intravenosa; Grupo 2 (G2) (controle) administração de adrenalina, sem realização de acupuntura. Nos dois grupos, foi administrada adrenalina, na dose inicial de $10 \mathrm{ug} / \mathrm{Kg}$, adicionando-se $5 \mathrm{ug} / \mathrm{Kg}$, a cada 5 minutos e finalizando em $40 \mathrm{ug} / \mathrm{Kg}$. Em todos os momentos avaliados foi realizado eletrocardiograma, comparando-se o número de registros de alterações em ambos os grupos. As principais alterações observadas foram ocorrência de bloqueio átrio-ventricular de 20 grau (BAV2) (G1: 92,3\% e G2: 100\%) e bradicardia (G1: $69,2 \%$ e G2: 92,3\%) nos 2 grupos. Verificou-se também ocorrência de taquicardia ventricular (TV) não sustentada (G1: 7,7\% e G2: $23,1 \%$ ), extra-sístoles (ES) isoladas (G1: $92,3 \%-14,5 \pm 11,7$ e G2:100\% - 24,9 13,9$)(p<0,05)$ e complexos

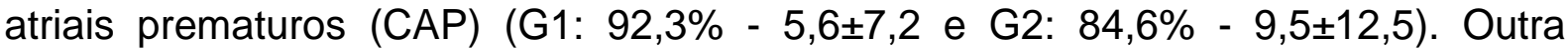
avaliação importante foi o tempo (em segundos) para restauração da frequência cardíaca a níveis fisiológicos (G1: 137,3 117,4 e G2: $253,5 \pm 151,1)(p<0,05)$ durante as ocorrências de BAV2 e bradicardia. Conclui-se que a acupuntura é eficaz em reduzir os episódios arrítmicos, além de atenuar os efeitos do reflexo de BezoldJarisch. Demonstrou-se que a acupuntura foi capaz de retardar a ocorrência de BAV2 e bradicardia e reduzir o tempo de recuperação para ritmo fisiológico, mostrando-se como ferramenta promissora na prevenção de arritmias cardíacas.
\end{abstract}

PALAVRAS-CHAVE: Acupontos. Adrenalina. Eletrocardiograma. PC6.C7

\section{COMPARATIVE STUDY OF THE ANALGESIC EFFECT OF KETAMINE $\mathbf{S}^{+}$ROUTE ORAL AND EPIDURAL IN BITCHES SUBMITTED TO OVARIOSALPINGOHISTERECTOMY}

\footnotetext{
ABSTRACT

This study evaluates acupuncture effect in the prevention of arrhythmias induced by epinephrine crescent doses in rats. Were studied 26 Wistar rats, healthy males divided into 2 groups: Group 1 (G1) - acupuncture for 30 minutes, points Pericardium ENCICLOPÉDIA BIOSFERA, Centro Científico Conhecer - Goiânia, v.11 n.22; p.2731 2015
} 
6 (Pc6) and Heart 7 (C7) before the intravenous administration of epinephrine, Group 2 (G2) - adrenaline, without performing acupuncture (control). In both groups, epinephrine was administered at an initial dose of $10 \mathrm{ug} / \mathrm{kg}$ by adding $5 \mathrm{ug} / \mathrm{kg}$ every 5 minutes until the final dose of $40 \mathrm{ug} / \mathrm{kg}$. In all periods evaluated electrocardiogram was performed by comparing the number of change records in both groups. It was observed that the main changes produced by adrenaline were the occurrence of atrioventricular block of second degree (BAV2) (G1: 92.3\% and G2: $100 \%$ ) and bradycardia (G1: $69.2 \%$ and G2: $92.3 \%$ ) in two groups. It has also occurrence of ventricular tachycardia (VT) not supported (G1: 7.7\% and G2: $23.1 \%$ ), extrasystole (ES) isolated (G1: $92.3 \%-14.5 \pm 117$ and G2: $100 \%-24.9 \pm 13.9)$ (p $<0.05)$ and premature atrial complexes (CAP) (G1: $92.3 \%-5.6 \pm 7.2$ and G2: $84.6 \%$ $-12.5 \pm 9.5$ ). Another important evaluation was the time (in seconds) for restoring the heart rate physiological levels (G1: $G 2$ and $137.3 \pm 117.4$ : $253.5 \pm 151.1)(p<0.05)$ during the occurrence of BAV2 and bradycardia. It was concluded that acupuncture is effective in reducing occurrences of ES, and mitigate the effects of the BezoldJarisch reflex, being able to delay the occurrence of bradycardia and BAV2 and reduce recovery time until physiological rhythm, showing up as a promising tool in the prevention of cardiac arrhythmias.

KEYWORDS: Acupoints. PC6. C7. Adrenaline. Electrocardiogram.

\section{INTRODUÇÃO}

As arritmias cardíacas são consideradas distúrbios na geração e/ou propagação do impulso elétrico, onde se observam anormalidades na origem, frequência e ritmo do mesmo (ETTINGER \& FELDMANN, 2004). Estudos que avaliam alternativas para correção das arritmias cardíacas são frequentemente realizados, necessitando de modelos experimentais que a induzam. Modelos cirúrgicos em ratos, que utilizam oclusão coronariana, são eficientes na obtenção de arritmias; entretanto estão associados à elevada mortalidade (ZORNOFF et al., 2009). Deste modo, modelos experimentais que usam a adrenalina em doses crescentes têm sido usados em cães (TEIXEIRA NETO et al., 2001; THIESEN et al., 2006), em porquinhos da Índia (HASHIMOTO, 2007) e em ratos (KAWAl et al., 2002).

A adrenalina é um agente simpatomimético de ação direta, sendo capaz de ativar todos os subtipos de receptores adrenérgicos. Sobre o sistema cardiovascular, provoca aumento do ritmo e débito cardíaco, do consumo de oxigênio e da irritabilidade das células marcapasso, levando a um maior risco de arritmias (HASHIMOTO, 2007).

A acupuntura é uma técnica da medicina tradicional chinesa que utiliza a estimulação de pontos específicos do corpo, chamados acupontos, para o tratamento de enfermidades (KOO et al., 2008). Estudos clínicos têm demonstrado que a acupuntura tem efeito terapêutico em isquemia do miocárdio e alguns tipos de arritmias (ZORNOFF et al., 2009). Os efeitos benéficos da acupuntura são atribuídos, em parte, pela inibição simpática (MIDDLEKAUFF et al., 2002). A acupuntura modula tônus de sistema autônomo, possibilitando correção de anormalidades na pressão sanguínea, através de alterações na frequência, contratilidade cardíaca e tônus vasomotor (LOPES, 2004).

Os detalhes de alguns dos mecanismos de ação da acupuntura ainda não são muito compreendidos e pesquisas em animais mostram, às vezes, descobertas contraditórias. Deste modo, o presente estudo teve como objetivo avaliar o efeito da 
acupuntura na prevenção de arritmias cardíacas induzidas por doses crescentes de adrenalina em ratos Wistar.

\section{MATERIAL E MÉTODOS}

Este estudo foi protocolado sob número 757 e cumpriu integralmente os Princípios Éticos na Experimentação Animal, adotados pela Sociedade Brasileira de Ciência em Animais de Laboratório (SBCAL). Foram utilizados 26 ratos (Rattus novergicus linhagem Wistar), hígidos, adultos, machos, com peso corporal entre 200 e 300 gramas, provenientes do Biotério Central da Instituição de origem. Durante o experimento os animais foram mantidos em gaiolas coletivas de polipropileno (5 animais/gaiola), recebendo água e ração comercial ad libitum, com controle de luz (ciclos de $12 \mathrm{~h}$ claro/escuro) e de temperatura $\left( \pm 25^{\circ} \mathrm{C}\right)$.

Os animais foram anestesiados com a associação de tiopental sódico (30 $\mathrm{mg} / \mathrm{Kg}$ ), via intraperitoneal (IP) e tiletamina-zolazepan $(10 \mathrm{mg} / \mathrm{Kg})$, administrado por via intramuscular (IM) (KANASHIRO \& CASSU, 2008). A seguir, foram distribuídos aleatoriamente em 2 grupos ( $n=13)$ : Grupo 1 (G1): submetido à acupuntura durante 30 minutos $(\mathrm{min})$ e, a seguir, induzida a arritmia com doses crescentes de adrenalina. Grupo 2 (G2): induzida a arritmia com doses crescentes de adrenalina, sem realização prévia de acupuntura (controle).

Nos ratos do G1, imediatamente após a anestesia, foi realizada a acupuntura manual, durante $30 \mathrm{~min}$, com agulhas de aço inox especiais para acupuntura, que foram inseridas bilateralmente nos pontos: Coração $7(\mathrm{C} 7)$, localizado proximal à articulação carpo-metacarpiana, abaixo do tendão flexor digital superficial, encontrado proximal à depressão triangular formada entre o tendão e o carpo; Pericárdio 6 (Pc6), localizado na face caudal, região proximal da articulação carpometacarpiana, proximal ao coxim acessório do carpo, entre os tendões flexor ulnar e flexor radial do carpo, que correm juntos (Fig. 1). Para estimulação dos pontos, as agulhas foram rotacionadas manualmente até o final do período de tratamento com a acupuntura (30 $\mathrm{min})$. Os ratos do G2 permaneceram em repouso durante 30 minutos, imediatamente após a anestesia, e foram utilizados como controle.

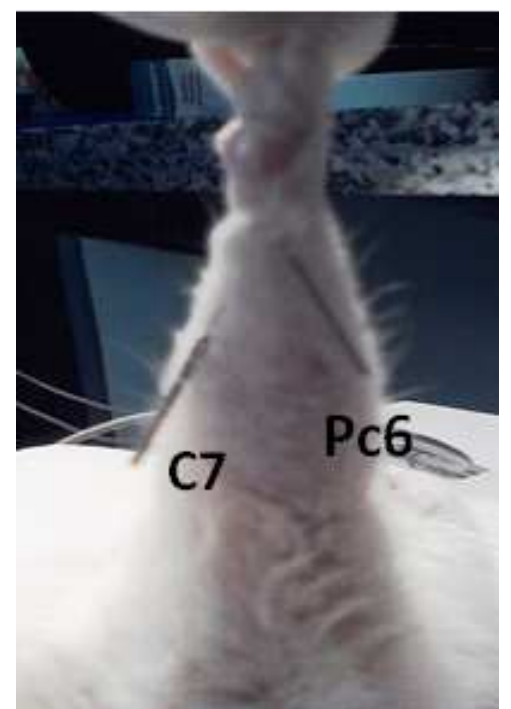

FIGURA 1 - Agulhas inseridas nos acupontos Coração 7 (C7) e Pericárdio 6 (Pc6) em membro anterior de rato. 
Iniciou-se então, em ambos os grupos, a indução de arritmias, realizada a partir do uso de adrenalina na dose inicial de $10 \mathrm{ug} / \mathrm{kg}$, via intravenosa (IV), pela canulação da veia peniana com cateter intravenoso periférico calibre 24G. A seguir, doses cumulativas de $5 \mathrm{ug} / \mathrm{kg}$ de adrenalina foram ministradas a cada $5 \mathrm{~min}$, pelo período de $35 \mathrm{~min}$, atingindo a dose máxima de 40ug $/ \mathrm{kg}$ (HASHIMOTO, 2007).

Antes e durante todo o período de administração da adrenalina foi realizado o registro do eletrocardiograma (ECG) nos momentos (M) a seguir descritos: M0: antes da administração de adrenalina; M1 (5 min): administração de $10 \mathrm{ug} / \mathrm{kg}$ de adrenalina; M2 (10 $\mathrm{min})$ : administração de $15 \mathrm{ug} / \mathrm{kg}$ de adrenalina; M3 (15 min): administração de $20 \mathrm{ug} / \mathrm{kg}$ de adrenalina; $\mathrm{M} 4$ (20 min): administração de $25 \mathrm{ug} / \mathrm{kg}$ de adrenalina; M5 (25 min) administração de $30 \mathrm{ug} / \mathrm{kg}$ de adrenalina; M6 (30 min) administração de $35 \mathrm{ug} / \mathrm{kg}$ de adrenalina; $M 7$ (35 min): administração de $40 \mathrm{ug} / \mathrm{kg}$ de adrenalina. Com os animais anestesiados, após a avaliação dos parâmetros do último momento (M7), procedeu-se a eutanásia dos animais por exsanguinação total, através de punção cardíaca.

Foi realizada eletrocardiografia computadorizada, com os animais posicionados em decúbito dorsal e os eletrodos fixados na região do olecrano em membros torácicos, e da patela em membros pélvicos, similar ao realizado em cães (ETTINGER \& FELDMAN, 2004). Registraram-se as derivações bipolares (I, II e III) e unipolares aumentadas (avR, avL e avF).

Foram avaliados: o ritmo cardíaco (sinusal, bradicardia sinusal, taquicardia ventricular não sustentada e bloqueio atrioventricular de segundo grau - BAV2), o número de ocorrências isoladas de extra-sístoles (ES) e complexos atriais prematuros (CAP) em cada momento avaliado, bem como a somatória da ocorrência de ES e CAP em cada um dos ratos estudados. Por ser o BAV2 a arritmia mais frequente do estudo, foi analisada a somatória do número de ocorrências da mesma, nos oito momentos avaliados ( $\mathrm{M} 0$ a M7), em cada um dos ratos. Além disto, avaliouse o tempo para restauração bradicardia nos ratos que apresentaram BAV2 e/ou bradicardia sinusal.

A análise estatística foi realizada com o programa SAS (Statistical Analysis System 9.1 - SAS Institute Inc., North Carolina, USA). A análise do ritmo cardíaco foi descritiva. Para comparação da somatória da ocorrência de ES, CAP e BAV2 nos oito momentos estudados, bem como do tempo de duração da bradicardia, entre os dois grupos, foi utilizado o teste de T não-pareado. Para comparação da ocorrência de ES, CAP e duração da bradicardia entre os diferentes momentos estudados dentro de cada um dos grupos, foi utilizada análise de variância (ANOVA), e para comparar estes parâmetros em cada um dos momentos avaliados, entre os 2 grupos, foi usado o teste de T não-pareado. Para comparar a ocorrência ou não de BAV2 (1- ausente; 2- presente) ao longo dos momentos (M0-M7), dentro de cada grupo, foi usado o Teste de Friedman, e para comparar esta variável em cada um dos momentos (M0 a M7), entre os 2 grupos, foi usado o teste de Mann-Whitney. Para todas as avaliações foi considerado nível de $5 \%$ de significância $(p<0,05)$.

\section{RESULTADOS E DISCUSSÃO}

Neste estudo empregou-se a rotação manual de Pc6 e C7, com tempo de inserção das agulhas de 30 minutos, e este pré-tratamento foi suficiente para inibir o aparecimento dos eventos arrítmicos supracitados, corroborando com outros autores que demonstraram que o tempo médio para obtenção de efeito cardiovascular com EA foi de 30 minutos após o início do tratamento (SYUU et al., 2003). 
Tradicionalmente é descrito um sistema de meridianos ou canais que conduzem energia pelo organismo e onde são distribuídos os acupontos (CASSU, 2002), passagem entre a pele e os meridianos de energia relacionados aos órgãos internos (SCHOEN, 2006). Estudos anteriores têm mostrado que Pc6 tem projeções para os centros cardíaco e vasomotor da medula, assim como para o segmento da coluna vertebral (da quinta vértebra cervical até a primeira torácica), que podem causar resposta simpática vascular (LI, 2001). SYUU et al. (1998) recomendam a utilização do ponto Pc6 para o tratamento de arritmias cardíacas em cães, enquanto STERNFELD (1989) indicam o uso do C7 para tratamento de arritmias supraventriculares não induzidas em pacientes humanos.

No momento inicial do estudo, que antecedeu a administração intravenosa de adrenalina (M0), todos os ratos apresentaram como único ritmo, o sinusal (Fig. 2I). Também, não foram observadas ES ou CAP neste momento, em ambos os grupos. $\mathrm{Na}$ avaliação do ritmo cardíaco no decorrer do estudo (M1 a M7), apenas um rato $(7,7 \%)$ do G1 não revelou qualquer distúrbio do mesmo, quando submetido às crescentes doses de adrenalina. Durante o experimento foram observados cinco tipos de alteração no ritmo cardíaco, sendo elas os BAV2, ES, taquicardia ventricular (TV) não-sustentada, CAP e bradicardia. Estes achados corroboram com os resultados de THIESEN et al. (2006) que também encontraram BAV2 e ES em cães submetidos a doses crescentes de adrenalina, anestesiados com isofluorano e prétratados com lidocaína. A presença destas alterações de ritmo reflete que a indução de arritmia com adrenalina em ratos no presente estudo foi efetiva.

Observou-se que a principal alteração produzida pela adrenalina sobre o ritmo cardíaco foi ocorrência de BAV2 (Fig. 2IIA e 2IIB) e bradicardia, nos dois grupos estudados. Treze ratos do grupo controle (G2) (100\%) e 12 do grupo acupuntura (G1) $(92,3 \%)$ apresentaram pelo menos uma ocorrência de BAV2, no decorrer dos 07 momentos avaliados (M1 a M7). A maioria dos ratos apresentou BAV2 no momento inicial dos bolus, evoluindo para bradicardia sinusal, que gradativamente cedeu até a normalização da frequência cardíaca (FC) e retorno ao ritmo sinusal.

Não foi observada diferença significante na somatória de ocorrência de BAV2 entre G2 e G1, que apresentaram médias de 5,1 $\pm 2,1$ e 3,5 $\pm 2,1$, respectivamente. Na evolução ao longo dos momentos, o G2 apresentou maior ocorrência de BAV2 em M4, M6 e M7, e o G1 em M2, M5, M6 e M7, todos em relação ao M0 (Tab. 1). Não foram observadas diferenças significantes quando comparados os dois grupos em cada um dos momentos do estudo.

Após a infusão de adrenalina, em consequência do mecanismo compensatório ao aumento da pressão, observa-se redução da FC e desenvolvimento de BAV2, arritmias que ocorrem devido a falha de propagação do impulso do átrio para o ventrículo e refletem uma anormalidade no nodo atrioventricular ou no sistema His-Purkinje (LASTE, 2001). TEIXEIRA NETO et al. (2001) também relataram o aparecimento de bradiarritmias, geralmente associadas a BAV2, em animais que receberam infusão de adrenalina. Outro estudo relatou aparecimento de BAV2 após a infusão de adrenalina em cães (THIESEN et al., 2006). Apesar de observar-se uma redução no número de BAV2 nos animais tratados com acupuntura neste estudo, não houve diferença significativa entre os grupos. Não foram encontrados na literatura outros estudos que correlacionassem a ocorrência de episódios de BAV2 em grupos tratados ou não com acupuntura. 
Valores de médias \pm desvios-padrão da duração da bradicardia (segundos) e medianas e percentis (P25; P75) da ocorrência de bloqueio atrioventricular de segundo grau (BAV2) dos ratos dos grupos acupuntura (G1) e controle (G2), submetidos a doses crescentes de adrenalina, avaliados nos oito momentos do estudo (M0 a M7).

\begin{tabular}{|c|c|c|c|c|c|c|}
\hline & & \multicolumn{3}{|c|}{ Duração de bradicardia } & \multicolumn{2}{|l|}{ BAV2 } \\
\hline & & G1 & & G2 & G1 & G2 \\
\hline Mo & & 0 & & 0 & $1(1 ; 1)$ & $1(1 ; 1)$ \\
\hline M1 & & $10,8 \pm 24,5$ & & $35,5 \pm 38,0$ & $1(1 ; 2)$ & $2(1 ; 2)$ \\
\hline M2 & & $20,5 \pm 26,1$ & ** & $45,8 \pm 53,5$ & $2(1 ; 2)^{*}$ & $2(1 ; 2)$ \\
\hline M3 & * & $25,8 \pm 36,9$ & * & $37,2 \pm 29,3$ & $2(1 ; 2)$ & $2(1 ; 2)$ \\
\hline M4 & ** & $28,1 \pm 34,5$ & & $34,8 \pm 32,3$ & $1(1 ; 2)$ & $2(1 ; 2)^{*}$ \\
\hline M5 & & $17,9 \pm 14,6$ & & $33,5 \pm 29,0$ & $2(1 ; 2)^{*}$ & $2(1 ; 2)$ \\
\hline M6 & - & $16,5 \pm 15,8$ & & $31,7 \pm 19,1$ & $1(1 ; 2)^{*}$ & $2(1 ; 2)^{*}$ \\
\hline M7 & * & $26,2 \pm 21,0$ & & $35,0 \pm 40,8$ & $2(1 ; 2)^{\star}$ & $2(1 ; 2)^{*}$ \\
\hline
\end{tabular}

${ }^{*}(p<0,05) \times M 0 ;{ }^{* *}(p<0,01) \times M 0 ; \bullet(p<0,05) \times G 2$. 1- ausente; 2- presente.

A bradicardia sinusal (Fig. 2IIIA) ocorreu em 12 ratos do G2 (92,3\%) e em 9 do G1 $(69,2 \%)$, sendo que a maioria das ocorrências foi consecutiva ao registro do BAV2. Durante os episódios de BAV2 e bradicardia, foi avaliado o tempo em segundos para restauração da bradicardia até níveis fisiológicos. A acupuntura foi capaz de reduzir o tempo de duração da bradicardia, em comparação com o controle. A duração média da bradicardia no $\mathrm{G} 1$ foi de $137,3 \pm 117,4$ enquanto no G2 foi de $253,5 \pm 151,1 \quad(p<0,05)$. Na evolução dos momentos, em cada um dos grupos, observou-se que os períodos de maior duração da bradicardia ocorreram em M2 e M3 (15 e 20ug/Kg de adrenalina) no G2, e em M3, M4 e M7 (20, 25 e $40 \mathrm{ug} / \mathrm{Kg}$ de adrenalina) no $\mathrm{G} 1$, todos em relação ao M0 (Tab. 1). Na comparação entre os dois grupos em cada um dos momentos, houve redução significativa da duração bradicardia no M6 do grupo acupuntura (G2), em relação ao Controle (G1).

A adrenalina é um fármaco agonista dos receptores alfa-adrenérgicos, localizados nos vasos periféricos, com ação vasoconstritora (LASTE, 2001). Tem como efeitos cardiovasculares o aumento da frequência cardíaca, da força de contração do coração e da pressão arterial (PA) e, através de reflexo vagal, induz a bradicardia reflexa. Durante a elevação da PA, nervos aferentes que compõem o sistema barorreflexo são estimulados (MARK \& DUNLAP, 2008) a partir de um estímulo sensorial proveniente dos pulmões e da parede do ventrículo esquerdo, desencadeando uma resposta eferente tônico-inibitória para o coração. As respostas culminam em apnéia e bradicardia, o que leva a redução do débito cardíaco e consequente hipotensão (ETTINGER \& FELDMANN, 2004). Esta tríade é observada na estimulação de reflexo cardiopulmonar conhecido como reflexo ENCICLOPÉDIA BIOSFERA, Centro Científico Conhecer - Goiânia, v.11 n.22; p.2736 
Bezold-Jarisch (MARK \& DUNLAP, 2008). Este fenômeno também foi relatado por TEIXEIRA NETO et al. (2001) e THIESEN et al. (2006), que verificaram bradicardia causada pela infusão contínua de adrenalina em cães anestesiados com halotano e isofluorano, respectivamente.

Com relação ao verificado no presente estudo, no que concerne à redução significante no tempo de duração da bradicardia reflexa em relação ao grupo controle e retardo no aparecimento de BAV2 e bradicardia, há concordância com estudos anteriores. LIN et al. (2010) relataram que o início da queda da frequência cardíaca em gatos anestesiados com quetamina, seguida por injeções intravenosas de $\alpha$-cloralose, foi adiada em aproximadamente 4 segundos no grupo tratado com acupuntura no ponto Pc6. Neste mesmo estudo, durante o estímulo com acupuntura manual por 10 minutos, o período de queda da FC foi diminuído em aproximadamente 10 segundos. Nos estudos de ZHOU et al. (2005), com 30 minutos de EA de baixa corrente e frequência, no ponto Jianshi-Neiguan (Pc5-6), houve decréscimo significativo na bradicardia reflexa em ratos. Os achados do presente estudo corroboram com a literatura citada.

Alguns estudos têm mostrado que a acupuntura modifica funções fisiológicas relacionadas com o sistema nervoso autônomo (MORI et al., 2002). MIDDLEKAUFF et al. (2002) demonstraram efeito inibitório da acupuntura sobre a atividade simpática em pacientes com insuficiência cardíaca em situações de estresse mental. WANG et al. (2002) relataram que a acupuntura aumenta a atividade vagal e diminui a atividade simpática em humanos saudáveis. $O$ aumento da atividade vagal produzido pela acupuntura, descrito pela literatura, é contrário ao observado no presente estudo, uma vez que o estímulo manual dos pontos Pc6 e C7 foi capaz de atenuar o reflexo de Bezold-Jarisch, observando-se redução da duração da bradicardia.

Outra alteração de ritmo presente no estudo, entretanto em frequência bem inferior ao BAV2 e a bradicardia, foi a taquicardia ventricular (TV) não sustentada (Fig. 2llIB) que ocorreu em três ratos do G2 (23,1\%) e em um do G1 $(7,7 \%)$. Estudos terapêuticos de arritmias não induzidas, em pacientes humanos com taquicardia supraventricular, demonstraram que, quando utilizados, os acupontos coração 7 (C7), pericárdio 6 (Pc6) e vaso concepção 17 (Vc 17) produziam efeito depressivo (STERNFELD, 1989). A acupuntura bilateral nos pontos Pc 6 e C7, foi eficiente para o tratamento de TV em equinos anestesiados com halotano, sendo também efetiva para redução do tempo de desaparecimento da TV após a infusão de dopamina (CÁRDENAS et al., 2009). Os resultados observados revelam que, independente da causa, protocolo anestésico ou tipo de estímulo empregado ao acuponto, o tratamento com acupuntura é considerado efetivo.

Neste estudo, a associação dos pontos Pc6 e C7 foi eficaz no controle das arritmias produzidas pela adrenalina, nos ratos avaliados. CÁRDENAS et al. (2009), ao avaliarem os efeitos da associação destes pontos, para tratamento de TV induzida por dobutamina em equinos anestesiados com halotano, descreve benefício no controle da TV, mas relata que não foi possível esclarecer se a associação dos pontos Pc6 e C7 apresentou um sinergismo favorável no tratamento da TV ou se o efeito isolado dos mesmos teria a mesma eficácia. De modo similar, o presente estudo não avaliou o estímulo isolado dos referidos acupontos e, assim, não se pode afirmar que a associação de ambos foi o fator responsável por uma resposta favorável na atenuação das arritmias.

Adicionalmente às alterações de ritmo observadas entre $M 1$ e $M 7$, foi registrada a presença de ES isoladas (Fig. 2IV) no ECG de todos os ratos do G2 
(100\%) e em 12 ratos do $\mathrm{G} 1$ (92,3\%), que apresentaram valores médios do número total desta ocorrência de $24,9 \pm 13,9$ e $14,5 \pm 11,7$, respectivamente, e redução significante no $G 1 \quad(p<0,05 \%)$. Esta redução, ocorrida no grupo tratado com acupuntura, sugere que ela foi capaz de inibir a formação deste tipo de distúrbio de condução. Nos momentos M1 e M2 houve redução da ocorrência de ES no G1, quando comparado ao G2. Na progressão dos bolus de adrenalina, não houve diferença entre os diferentes momentos no G1, enquanto o G2 apresentou maior ocorrência de ES em M1, em relação ao M0 e M7 (Tab. 2). Estudos têm mostrado que a EA em acupontos localizados ao longo dos nervos somáticos profundos, como o nervo mediano (Pc6) ou o nervo fibular profundo (St36), reduz a resposta simpatoexcitatória e a frequência de extra-sístoles ventriculares (GUO et al., 1984).

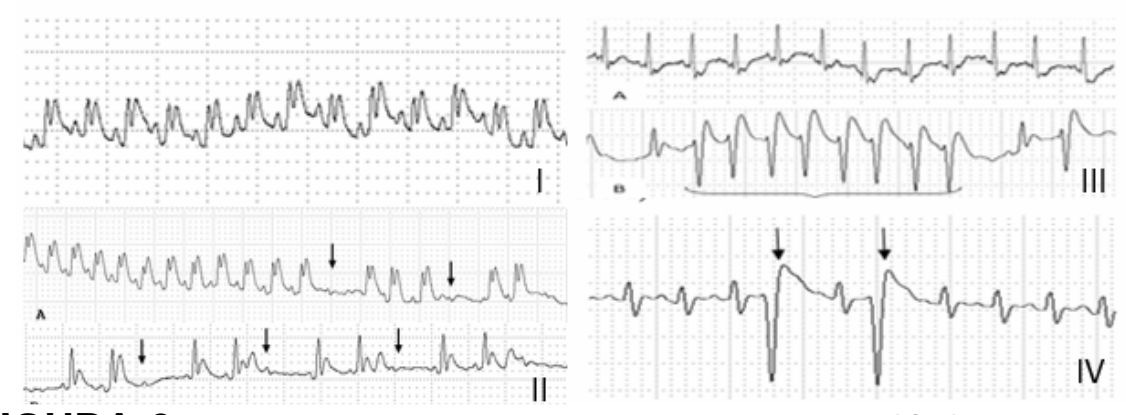

FIGURA 2 - I. Ritmo sinusal registrado no rato 14 (G2) em M4. A) Início do BAV2 (Seta) no rato 20 do G2 em M5. B) BAV2 com várias ondas $p$ bloqueadas (setas) no rato 80 do $\mathrm{G} 1$ em M3. III. A) Bradicardia sinusal, registrada no rato 14 do G2 em M6. B) Episódio de taquicardia ventricular não sustentada $\left(^{*}\right)$ no rato 35 do G2 em M1. IV. Extrasístoles isoladas no rato 27 do G2 (M3). Derivação II. 2N. $25 \mathrm{mmseg}$.

TABELA 2 - Médias e desvios-padrão dos parâmetros número de ocorrências de extra-sístoles (ES) e número de complexos atriais prematuros (CAP), dos ratos dos grupos acupuntura (G1) e controle (G2), avaliados nos oito momentos do estudo (M0 a M7).

\begin{tabular}{lllll}
\hline & ES & \multicolumn{3}{c}{ CAP } \\
\hline & G1 & G2 & G1 & G2 \\
\hline M0 & 0 & 0 & 0 & 0 \\
M1 & $2,3 \pm 4,3^{*}$ & $7,8 \pm 7,1$ & $0,1 \pm 0,3$ & $1,1 \pm 2,4$ \\
M2 & $1,1 \pm 1,9 * *$ & $3,2 \pm 2,9$ & $0,4 \pm 0,9$ & $0,69 \pm 1,3$ \\
M3 & $2,0 \pm 2,7$ & $4,3 \pm 3,9$ & $0,6 \pm 1,1$ & $1,3 \pm 3,0$ \\
M4 & $1,8 \pm 2,6$ & $5,1 \pm 11,3$ & $0,5 \pm 1,4$ & $0,5 \pm 1,1$ \\
M5 & $2,0 \pm 2,6$ & $1,9 \pm 1,5$ & $0,7 \pm 1,4$ & $1,7 \pm 3,2$ \\
$M 6$ & $2,7 \pm 4,11$ & $1,5 \pm 1,2$ & $0,6 \pm 1,5$ & $1,1 \pm 3,0$ \\
$M 7$ & $3,5 \pm 10,42$ & $1,1 \pm 2,1$ & $1,3 \pm 2,3$ & $0,1 \pm 0,3$ \\
\hline${ }^{*}(p<0,01) \times G 2 ;{ }^{* *}(p<0,05) \times G 2 ; \sim(p<0,05) \times M 0 ; \bullet(p<0,05) \times M 7$ &
\end{tabular}


As contrações cardíacas prematuras (CAP) são complexos que se originam fora do nó-sinusal e interrompem o ritmo cardíaco normal (TEIXEIRA NETO et al., 2001). Ocorrência de CAP em G2 e G1 também foi notada, com médias de 9,5 $\pm 12,5$ e 5,6 $\pm 7,2$, respectivamente. Apenas um animal do G1 $(7,7 \%)$ e dois do $G 2(15,4 \%)$ não apresentaram nenhuma ocorrência de CAP ao longo do estudo. $\mathrm{Na}$ comparação entre os dois grupos, em cada momento, bem como entre os momentos dentro de cada grupo, não foram observadas diferenças significantes. Os valores de médias e desvios-padrão da ocorrência de CAP em todos os momentos estão descritos na Tabela 2.

Apesar de não haver diferença estatística, pode-se observar que os animais submetidos à acupuntura obtiveram redução no número de ocorrências de CAP. Na literatura consultada, não foram encontrados dados sobre os efeitos da acupuntura na ocorrência de CAP. Desta forma, é importante que sejam realizados mais estudos a fim de investigar os efeitos da acupuntura no complexo atrial prematuro.

O uso da acupuntura como terapia complementar está bem estabelecido, porém o desenvolvimento de pesquisas no campo da acupuntura é muito importante, pois apesar da eficácia demonstrada em várias situações clínicas, a carência nas bases científicas e a falta de conhecimento dos mecanismos pelos quais ocorrem os efeitos da acupuntura podem restringir seu uso. De acordo com o exposto neste estudo, pode-se perceber que os efeitos da acupuntura não podem ser explicados por um mecanismo simples. Um estímulo que começa como um evento local difunde-se pelo sistema nervoso, cria alterações endócrinas e imunológicas, afetando o organismo como um todo. Assim, são necessários ainda mais estudos avaliando a função da acupuntura na fisiologia cardiovascular e os mecanismos subjacentes, para analisar se a acupuntura pode reduzir a incidência ou gravidade de arritmias.

\section{CONCLUSÃO}

O estímulo manual dos acupontos Pc6 e C7 é eficaz como pré-tratamento à prevenção de arritmias induzidas pela infusão de doses crescentes de adrenalina em distúrbios do ritmo cardíaco. Os dados demonstram que houve redução na ocorrência de bloqueios atrioventriculares de $2^{\circ}$ grau, extrassístoles, complexos atriais prematuros e taquicardia ventricular não sustentada, além da atenuação dos efeitos do reflexo de Bezold-Jarisch.

\section{REFERÊNCIAS}

CÁRDENAS, J.; LUNA, S.P.L.; TEIXEIRA NETO, F; BEIER, S.L. Comparação entre a lidocaína e a acupuntura no tratamento da taquicardia ventricular induzida com dopamina em equinos anestesiados com halotano. Arquivo Brasileiro de Medicina Veterinária e Zootecnia, v.61, n.4, p.777-784, 2009.

CASSU, R. N. Avaliação dos efeitos cardiorrespiratório, endócrino e analgésico da eletroacupuntura em cães. 2002. 197f. Tese (Doutorado em Anestesiologia) Faculdade de Medicina da Universidade Estadual Paulista, Botucatu, 2002.

ETTINGER, S. J.; FELDMAN, E. C. Tratado de Medicina Interna Veterinária. 5. Ed. V. 1.Rio de Janeiro: Guanabara Koogan, 2004. 1041 p. 
GUO, X.Q.; XIA, Y.; LI, P. Role of arcuatus area in inhibitory action of somatic nerve stimulation on ventricular extrasystoles induced by hypothalamic stimulation in rabbits. Acta Physiologica Sínica, v.36, p.9-15, 1984.

HASHIMOTO, K. Arrhythmia models for drug research: classification of antiarrhythmic drugs. Journal of Pharmacological Sciences, v.103, n.4, p.333-346, 2007.

KANASHIRO, G. P.; CASSU, R. N. Anestesia em Animais de Laboratório. In: ANDRADE, S. F. Manual de Terapêutica Veterinária. 3ed. São Paulo: Roca, p. 727-745.2008.

KAWAI, Y.; HAYASHI, Y.; ITO, I.; YASHI, T.K.; TAKADA, K.; KAGAWA, K.; YAMATODANI, A.; MASHIMO, T. Nicorandil prevents epinephrine-induced arrhythmias in halothane-anesthetized rats by nitric oxide-dependent mechanism. Archives Pharmacology, v.366, p.522-7, 2002.

KOO, S.T.; LIM, K.S.; CHUNG, K. Electroacupuncture-induced analgesia in a rat model of ankle sprain pain is mediated by spinal alpha-adrenoceptors. Pain, v.135, p.11-19, 2008.

LASTE, N. J. Cardiovascular pharmacotherapy. Veterinary Clinics of North America: Small Animal Practice, v.31, n.6, p.1231-1254, 2001.

LI, P. The anatomical and physiological rule of acupoints. In: CHEUNG, L.; LI, P.; WONG, C. The Mechanism of Acupuncture Therapy and Clinical Case Studies. New York: Taylor and Francis, 2001.

LIN, J.H.; SHIH, C.H.; KAPHLE, K.; WU, L.S.; TSENG, W.Y.; CHIU, J.W.; LEE, T.C.; WU, Y.L. Acupuncture Effects on Cardiac Functions Measured by Cardiac Magnetic Resonance Imaging in a Feline Model. Evidence-Based Complementary Alternative Medicine, v.7, n.2, p.169-76, 2010.

LOPES, T.F.T. Acupuntura no tratamento de arritmias cardíacas. 2004. $38 f$. Monografia (Especialização Latu sensu) - Faculdade de Medicina Veterinária, Universidade Estadual Paulista, Botucatu, 2004.

MARK, E.; DUNLAP, M.D. Cardiopulmonary baroreflexes. Hypertension primer A39, p.123-125, 2008.

MIDDLEKAUFF, H.R.; HUI, K.; YU, J.L.; HAMILTON, M.A.; FONAROW, G.C.; MORIGUCHI, J.; MACLELLAN, W.R.; HAGE, A. Acupuncture inhibits sympathetic activation during mental stress in advanced heart failure patients. Journal of Cardiac Failure, v.8, p.399-406, 2002.

MORI, H.; NISHIJO, K.; KAWAMURA, H.; ABO, T. Unique immunomodulation by electro-acupuncture in humans possibly via stimulation of the autonomic nervous system. Neuroscience Letters, v.320, p.21-24, 2002. 
SCHOEN, A. Acupuntura veterinária da arte antiga à medicina moderna. 2.ed. São Paulo: Roca, p.91-108.2006.

STERNFELD, M. Acupunture \& Supraventricular tachicardia. American Journal of Acupuncture, v.17, p.119-24, 1989.

SYUU, Y.; MATSUBARA, H.; HOSOGI, S.; SUGA, H.. Pressor effect of electroacupuncture on hemorrhagic hypotension. American Journal of Physiology - Regulatory, Integrative and Comparative Physiology, v.285, p.1446-1452, 2003.

SYUU, Y.; MORI, S.; TAKESHI, M.; JUN'ICHI, A.; HIDEO, S.; HIROYUKI, S. Effects of acupuncture on Cardiovascular Function. Circulation Control, v. 19, n.04, p. 509518, 1998.

TEIXEIRA NETO, F.J.; MASSONE, F.; LUNA, S.P.; CAMACHO, A.A.; ISHIY, H.M. The effects of atropine and methotrimeprazine on the epinephrine-induced arrhythmias in halothane-anesthetized dogs. Canadian Journal of Veterinary Research, v.65, p.116-124, 2001.

THIESEN, R.; NUNES, N.; BELMONTE, E. A.; BARBOSA, V. F.; CONCEIÇÃO, E. D. V.; MORO, J. V. Efeitos cardiovasculares da infusão contínua de lidocaína em cães anestesiados com isofluoranoe submetidos a doses crescentes de adrenalina. Arquivo Brasileiro de Medicina Veterinária e Zootecnia, v.64, n.1, 2006.

WANG, J. KUO, T. YANG, C. An alternative method to enhance vagal activities and suppress sympathetic activities in humans. Autonomous Neuroscience 100, 90-95, 2002.

ZHOU, W.; FU, L.W.; LOI, S.C.T.; LI, P.; LONGHURST, C. Afferent mechanism underlying stimulation-modality related modulation of acupuncture-related cardiovascular response. Journal of Applied Physiology, v.98, p.872-880, 2005.

ZORNOFF, L.A.M.; PAIVA, S.A.R.; MINICUCCI, M.F.; SPADARO, J. Infarto do Miocárdio Experimental em Ratos: Análise do Modelo. Arquivos Brasileiros Cardiologia, v.93, n.4, p.434-440, 2009. 